\title{
Low-energy (100 GeV - few TeV) neutrino point source searches in the southern sky with IceCube
}

\author{
The IceCube Collaboration ${ }^{\dagger}$ \\ ${ }^{\dagger}$ http://icecube.wisc.edu/collaboration/authors/icrc15_icecube \\ E-mail: rickard.stromephysics.uu.se
}

IceCube searches for neutrino point sources in the southern sky have traditionally been restricted to energies well above $100 \mathrm{TeV}$, where the background of down-going atmospheric muons becomes sufficiently low to be tolerated in searches. Recent developments of a data stream dedicated to the study of low-energy neutrinos from the Southern Hemisphere enable searches to be extended far below this threshold. This data stream utilizes powerful veto techniques to reduce the atmospheric muon background, allowing IceCube for the first time to perform a search for point-like sources of neutrinos in the southern sky at energies between $100 \mathrm{GeV}$ and a few TeV. We will present the event selection along with the results obtained using one year of data from the full detector.

Corresponding authors: R. Ström ${ }^{1 *}$,

${ }^{1}$ Department of Physics and Astronomy, Uppsala University, Box 516, 75120 Uppsala, Sweden

The 34th International Cosmic Ray Conference,

30 July- 6 August, 2015

The Hague, The Netherlands

${ }^{*}$ Speaker. 


\section{Introduction}

Neutrinos interact through gravity and the weak interaction only and can therefore, unlike other messenger particles like photons and protons, travel astronomical distances without experiencing significant absorption or deflection in magnetic fields. Further, they constitute a unique probe for distances larger than $50 \mathrm{Mpc}$ and from extremely dense environments. This makes neutrinos ideal as cosmic messengers that can be used to explore the most powerful accelerators in the Universe, in particular the mechanisms for producing and accelerating cosmic rays to incredible energies.

By studying clustering of neutrino candidate events in the IceCube Neutrino Observatory (IceCube) we can discover sites of hadronic acceleration where neutrinos are produced in the decay of charged pions created when primary cosmic ray protons and nuclei interact with radiation and matter in the vicinity of the acceleration site.

The Southern Hemisphere is particularly interesting, containing both the Galactic Center and the main part of the Galactic plane. For IceCube this means we are studying down-going tracks in a much larger background of muons created as cosmic rays enter the Earth's atmosphere. The traditional workaround has been to study only the highest energy neutrinos well above $100 \mathrm{TeV}$, where the background of atmospheric muons is sufficiently low. In the LESE (Low Energy Starting Events) analysis presented in this contribution we have lowered the energy threshold significantly utilizing several veto techniques, i.e. by looking for starting events in the detector. In particular we study neutrinos from soft spectra with cut offs in $\mathrm{TeV}$-scale as observed for Galactic $\gamma$-ray sources, see e.g. [1, 2, 3], and references therein.

\section{Data and Simulation}

IceCube instruments a cubic-kilometer of ice and is the world's largest neutrino telescope, located under the ice cap of the geographic South Pole, Antarctica [4]. It consists of 5160 optical modules deployed on 86 cables, called strings, and arranged in a three-dimensional grid between 1450 and $2450 \mathrm{~m}$ beneath the surface. The ultra pure ice at such depths is ideal for observations of Cherenkov light from charged leptons created when neutrinos interact with molecules in the ice or in the nearby bedrock.

Each optical module operates as an individual detector, a so-called DOM (Digital Optical Module), and consists of a photomultiplier tube, digitizer electronics and calibration LEDs, all housed in a pressure-resistant glass sphere. When two neighboring or next-to-neighboring DOMs on the same string both cross the 0.25 photoelectron discriminator threshold of the PMT within a $1 \mu \mathrm{s}$ time window, the signals qualify to be in Hard Local Coincidence (HLC). The leading trigger condition is formed when at least eight DOMs with HLCs trigger within a $5 \mu$ s time window. In this analysis we also use events from a trigger dedicated to catching almost vertical events as well as one with a lower effective energy threshold active in a denser central subarray called DeepCore [5]. The total trigger rate considered is around $2.8 \mathrm{kHz}$. The data is reduced via a series of straight cuts dedicated to reconstruction and calorimetric quality and a cut on an event score calculated using a machine learning algorithm.

We present the analysis and results for data taken between May 2011 and May 2012, the first year of the completed 86-string IceCube detector. Runs were carefully selected to ensure that the 

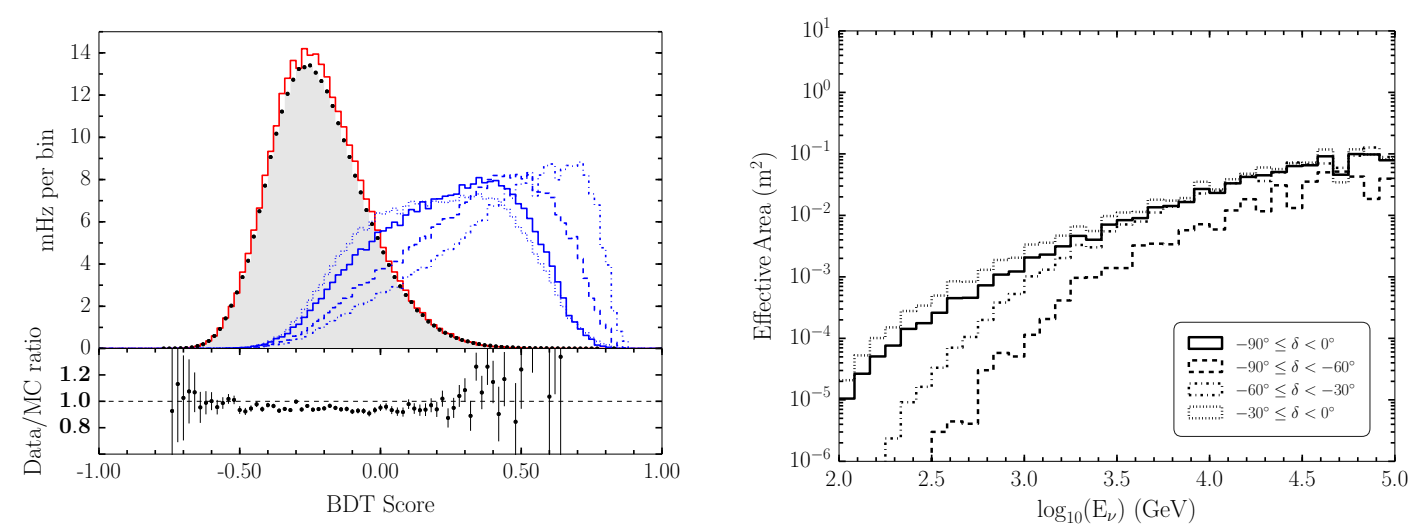

Figure 1: Left: BDT score distribution where experimental data is shown in black dots with error bars showing the statistical uncertainty (too small to be seen on this scale) and is further illustrated by the gray shaded area. Simulated atmospheric muons are presented in the solid red line. Signal hypotheses are shown in blue lines normalized to experimental data: $\mathrm{E}_{v}^{-2}$ (dash-dotted), $\mathrm{E}_{v}^{-2} e^{-\mathrm{E}_{v} / 10 \mathrm{TeV}}$ (dashed), $\mathrm{E}_{v}^{-2} e^{-\mathrm{E}_{v} / 1 \mathrm{TeV}}$ (solid) and $\mathrm{E}_{v}^{-3}$ (dotted). Simulated signals are defined as starting inside the instrumented detector volume. Right: Neutrino effective area as a function of neutrino energy.

majority of the DOMs used in vetoes were active, by both requiring a certain fraction of active DOMs overall and individually in the two different veto regions applied at the filter level, but also by studying the event rates at higher analysis levels. The final livetime considered is 329 days. The true right ascension directions of the data were kept blind until the last step of the analysis to keep the event selection and the final likelihood implementation as unbiased as possible.

\section{Selecting Low-Energy Starting Events}

The event selection is optimized for neutrinos in the energy region between $100 \mathrm{GeV}$ to a few $\mathrm{TeV}$. The outermost parts of the instrumented detector are used as a veto towards incoming muons. The triggered data is reduced by both real-time filtering at the South Pole, as well as subsequent offline CPU-intensive processing.

The development of a real-time full sky filter with a low energy threshold was crucial to reach lower energies, since many of the traditional IceCube real-time filters for the Southern Hemisphere were effectively using a charge cut, focusing on keeping events from $100 \mathrm{TeV}$ and up. The filter consists of two steps: a hit pattern based part, that rejects events with recorded hits in any of the 5 top DOMs or with the first HLC hit on any of the outermost strings, followed by a rejection of events with a reconstructed interaction vertex outside of the fiducial volume ${ }^{1}$. About $170 \mathrm{~Hz}$ pass the filter and is sent North for offline processing.

To ensure that we have enough information in the events for advanced angular and energy reconstructions we begin by considering overall quality cuts, such as a minimum required number of photoelectrons. We further reject events with a poor reconstruction quality. Several advanced vetoes are used to reduce the number of muons leaking in. In particular we are studying causality

\footnotetext{
${ }^{1}$ A polygon shaped region defined by connecting lines that go through points at $90 \%$ of the distance from the center string (string 36) to the outermost strings at the position of the 8 corners of the detector (as seen from the top) and the region below the 5 topmost DOMs.
} 

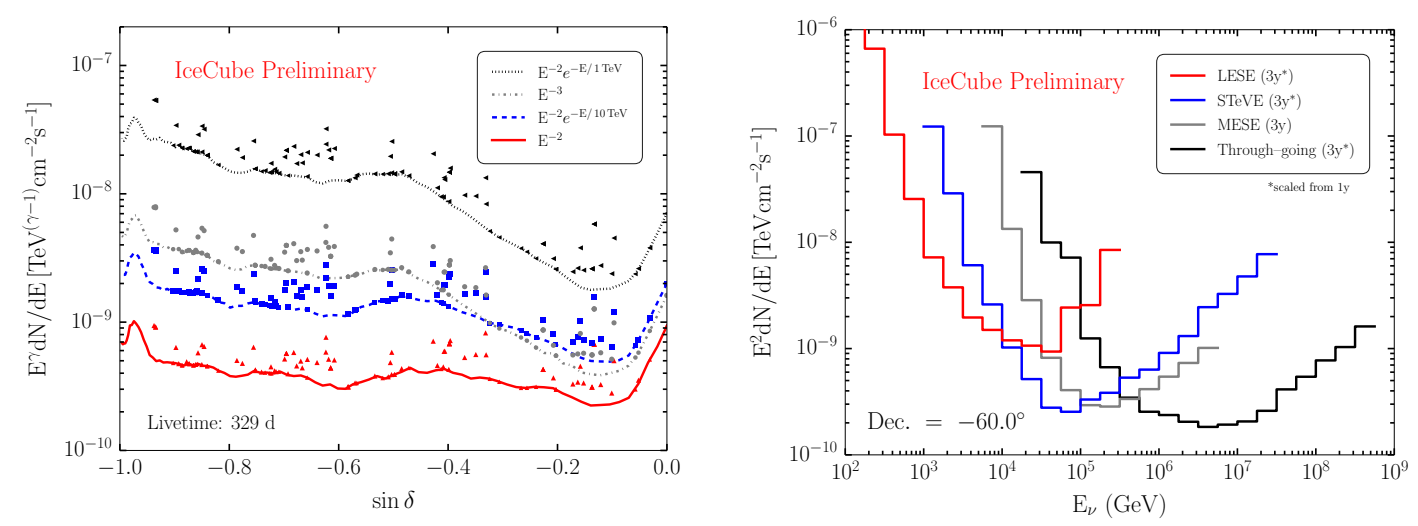

Figure 2: Left: Median sensitivities as a function of declination (line) and source upper limits (markers) at $90 \%$ C.L. Right: Differential median sensitivity corresponding to 3 years of operation (at $-60^{\circ}$ and $90 \%$ C.L.). Created by simulating point sources with an $\mathrm{E}^{-2}$ spectrum over quarter-decades in energy.

of hits in an extended veto region, consisting both of the outer regions of IceCube and the IceTop surface array [6].

As a major step in the event selection we apply a machine learning algorithm: a Boosted Decision Tree (BDT) is programmed to exploit the remaining separation between signal and background. In particular, it focuses on directional and calorimetric information. The BDT event score is shown in figure 1 . We make a cut at 0.40 which was found to be optimal or close to optimal for a wide range of signal hypotheses.

The angular reconstruction uncertainty $\sigma$ is estimated individually for each event as $\sigma^{2}=$ $\left(\sigma_{1}^{2}+\sigma_{2}^{2}\right) / 2$ where $\sigma_{1,2}$ are the major/minor axes of an ellipse that constitute the conic section of a paraboloid fitted to the likelihood space around the reconstruction minimum [7]. The ellipse is located at a level corresponding to $1 \sigma$ indicating $68 \%$ containment. This quantity often underestimates the angular uncertainty, which is why we apply an energy dependent correction to the pull as seen in simulation, defined as the ratio of the true reconstruction error to the estimated reconstruction error, based on the median angular resolution. Events with a corrected value above $5^{\circ}$ were rejected after optimization studies of the final sensitivity. The median angular resolution of the signal sample is about $2^{\circ}$.

The energy loss pattern for each event is estimated using an algorithm fitting hypothetical cascade-like energy depositions along the input track reconstruction [8]. An energy proxy for the neutrino energy is constructed by adding the contributions that are reconstructed inside the instrumented volume.

The final sample consists of 6191 events, a rate of about $0.2 \mathrm{mHz}$, and is still dominated by atmospheric muons leaking in through the veto layers, effectively mimicking starting events. The effective area for the full southern sky as well as in three declination bands is shown in figure 1.

\section{Search for Clustering of Events}

We look for point sources by searching for spacial clustering of events in the Southern Hemisphere. Hypotheses are tested using an unbinned maximum likelihood method [9] where the events 
in the final sample contribute individually with their reconstructed position $\mathbf{x}_{i}$, reconstructed energy $E_{i}$ and an estimation of the angular uncertainty $\sigma_{i}$. The likelihood $\mathcal{L}$ is a function of two fit parameters: $n_{s}$ (the number of signal events) and $\gamma$ (spectral index of a source $S$ located at position $\mathbf{x}_{S}$ with an unbroken power law spectrum) and contains the probability for an event to be signalor background-like. The final likelihood function is a sum over $N$ events and is maximized with respect to $n_{s}$ and $\gamma$ where $n_{s}$ is constrained to be non-negative:

$$
\mathcal{L}\left(n_{s}, \gamma\right)=\prod_{i}\left(\frac{n_{s}}{N} \mathcal{S}_{i}\left(\mathbf{x}, \mathbf{x}_{S} ; \sigma, E, \gamma\right)+\left(1-\frac{n_{s}}{N}\right) \mathcal{B}_{i}(\boldsymbol{\delta} ; E)\right) .
$$

The signal PDF $\mathcal{S}$ contains a spacial part parametrized with a two-dimensional gaussian profile with extension $\sigma_{i}$ and since Icecube, due to its location, has a uniform acceptance in right ascension, the spacial part of the background PDF $\mathcal{B}$ is represented with a spline fit to the full sample of experimental data depending on the declination $\delta$ only. The calorimetric parts of these PDFs consist of two-dimensional spline fits to the reconstructed energy $E_{i}$ and declination $\delta_{i}$.

The test statistic $\mathcal{T S}$ used is the likelihood ratio $\Lambda$ taken with respect to the null hypothesis $\left(n_{s}=0\right)$ :

$$
\mathcal{T S}=2 \log \Lambda=\sum_{i} \log \left(1+\frac{n_{s}}{N}\left(\frac{\mathcal{S}_{i}}{\mathcal{B}_{i}} \mathcal{W}_{i}-1\right)\right) \equiv \sum_{i} \log \left(1+n_{s} \chi_{i}\right),
$$

where $\chi_{i}$ is the individual event weight evaluated for a point source hypothesis at a particular location.

An all sky search is performed looking for an excess of neutrino candidate events in the Southern Hemisphere (declination range $-85^{\circ}$ to $0^{\circ}$ ) on a HEALPix ${ }^{2}$ [10] grid corresponding to a resolution of about $0.5^{\circ}$. In a second iteration we zoom in on the most interesting regions. This search is not motivated by any prior knowledge regarding the position of the sources, instead we are limited by the large number of trials performed, which together with the angular resolution gives a limit on the effective number of individual points tested. The pre-trial p-values obtained are converted into post-trial p-values by counting the fraction of times we get an equal or greater pre-trial p-value from an ensemble of 10000 pseudo-experiments where the right ascension directions were scrambled, see figure 3.

We also perform a search at the position of 96 pre-defined sources: all $84 \mathrm{TeVCat}^{3}$ [11] sources in the Southern Hemisphere (as of May 2015) and in addition 12 sources traditionally tested by IceCube ('GX 339-4', 'Cir X-1', 'PKS 0426-380', 'PKS 0537-441', 'QSO 2022-077', 'PKS 1406-076', 'PKS 072711', 'QSO 1730-130', 'PKS 0454-234', 'PKS 1622-297', 'PKS 1454-354', 'ESO 139-G12'). The TeVCat listing consists of sources seen by ground-based gamma-ray experiments, such as VERITAS, MAGIC, and H.E.S.S. and from these we consider sources from the catalogs 'Default Catalog' and 'Newly Announced', stable sources published or pending publication in refereed journals.

Figure 2 shows the median sensitivity of the LESE analysis for four different signal hypotheses as a function of declination. Further we illustrate the joint effort within the IceCube collaboration towards lowering the energy threshold for point source searches by showing the differential sensitivity for four different analyses: the LESE analysis, the STeVE (Starting TeV Events) analysis optimized at slightly higher energies (few $\mathrm{TeV}-100 \mathrm{TeV}$ ) [12], and the classic through-going point

\footnotetext{
${ }^{2} \mathrm{http}: / /$ healpix.sourceforge.net

${ }^{3}$ http://tevcat.uchicago.edu
} 

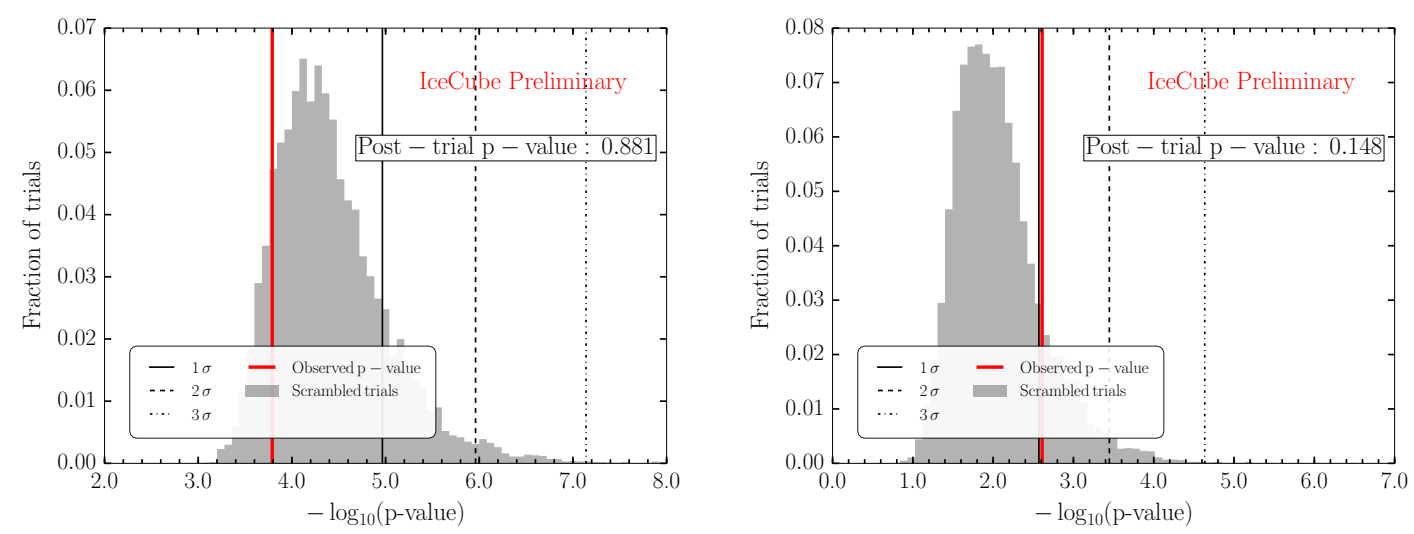

Figure 3: Pre-trial p-value distribution from 10000 scrambled trials for the sky scan (left) and the source list scan (right). The red line indicates the observed best $p$-values for the hottest spot and hottest source respectively, while the black vertical lines represent the 1,2 , and $3 \sigma$ significance levels.

source analysis in black [13]. The sensitivities shown correspond to 3 years of operation and where estimated by simulating events with the livetime corresponding to the MESE (Medium-Energy Starting Events) analysis focusing on energies in the region $100 \mathrm{TeV}-1 \mathrm{PeV}$ [14].

\section{Results}

The pre-trial p-value distribution for the all sky search is shown in figure 4 . The hottest spot is located at right ascension $305.2^{\circ}$ and declination $-8.5^{\circ}$ with a pre-trial p-value of $1.6 \cdot 10^{-4}$ and a post-trial p-value of $88.1 \%$ (best fit parameters $\hat{n}_{s}=18.3$ and $\hat{\gamma}=3.5$ ). In figure 5 we show a zoom in on the $\mathcal{T S}$ distribution in the area surrounding the hottest spot and further a zoom in on the individual event weights $\log \left(1+n_{s} \chi_{i}\right)$ that contribute to the test statistic at the position and with the best fit parameters of the hottest spot.

The most significant source in the pre-defined list was QSO 2022-077 ${ }^{4}$, a Flat-Spectrum Radio Quasar (FSRQ), with a pre-trial p-value of $2.5 \cdot 10^{-3}$. The post-trial p-value is $14.8 \%$. For the sources in the list, we calculate the upper limit at $90 \%$ confidence level (C.L.) based on the classical approach [15] for the signal hypotheses: $d \Phi / d \mathrm{E}_{v} \propto \mathrm{E}_{v}^{-2}, \mathrm{E}_{v}^{-2} e^{-\mathrm{E}_{v} / 10 \mathrm{TeV}}, \mathrm{E}_{v}^{-2} e^{-\mathrm{E}_{v} / 1 \mathrm{TeV}}$, and $\mathrm{E}_{v}^{-3}$,

Table 1: The most significant sources in the pre-defined list. The p-values are the pre-trial probability of being compatible with the background-only hypothesis. The $\hat{n}_{s}$ and $\hat{\gamma}$ are the best-fit values from the likelihood optimization. We further show the flux upper limit $\Phi$ at $90 \%$ C.L. for a number of neutrino flux models. These are shown for the combined flux of $v_{\mu}$ and $\bar{v}_{\mu}$ in units $\mathrm{TeV}^{\gamma-1} \mathrm{~cm}^{-2} \mathrm{~s}^{-1}$.

\begin{tabular}{|c|c|c|c|c|c|c|c|c|c|}
\hline Source & R.A. $\left[{ }^{\circ}\right]$ & $\operatorname{dec} .\left[^{\circ}\right]$ & $-\log _{10}$ (p-val.) & $\hat{n}_{s}$ & $\hat{\gamma}$ & $\mathrm{E}_{v}^{-2}$ & $\begin{array}{r}\Phi_{v_{\mu}+\bar{v}_{\mu}}^{99 \%} \times \mathrm{T} \\
\mathrm{E}_{v}^{-2} e^{-\mathrm{E}_{v} / 10 \mathrm{TeV}}\end{array}$ & $\begin{array}{l}v^{\gamma-1} \mathrm{~cm}^{-2} \mathrm{~s}^{-1} \\
\mathrm{E}_{v}^{-2} e^{-\mathrm{E}_{v} / 1 \mathrm{TeV}}\end{array}$ & $\mathrm{E}_{v}^{-3}$ \\
\hline QSO 2022-077 & 306.5 & -7.6 & 2.61 & 17.3 & 3.5 & $6.74 \cdot 10^{-10}$ & $5.81 \cdot 10^{-9}$ & $1.56 \cdot 10^{-9}$ & $1.27 \cdot 10^{-9}$ \\
\hline HESS J1718-385 & 259.5 & -38.5 & 1.58 & 5.9 & 3.4 & $7.62 \cdot 10^{-10}$ & $3.21 \cdot 10^{-8}$ & $2.80 \cdot 10^{-9}$ & $5.58 \cdot 10^{-9}$ \\
\hline HESS J1841-055 & 280.3 & -5.53 & 1.48 & 5.0 & 2.4 & $5.01 \cdot 10^{-10}$ & $4.52 \cdot 10^{-9}$ & $1.19 \cdot 10^{-9}$ & $9.88 \cdot 10^{-10}$ \\
\hline KUV 00311-1938 & 8.4 & -19.4 & 1.40 & 7.1 & 3.4 & $8.25 \cdot 10^{-10}$ & $1.33 \cdot 10^{-8}$ & $2.47 \cdot 10^{-9}$ & $2.65 \cdot 10^{-9}$ \\
\hline 30 Dor $\mathrm{C}$ & 83.9 & -69.1 & 1.04 & 2.3 & 3.6 & $9.14 \cdot 10^{-10}$ & $5.35 \cdot 10^{-8}$ & $3.65 \cdot 10^{-9}$ & $7.92 \cdot 10^{-9}$ \\
\hline
\end{tabular}

\footnotetext{
${ }^{4}$ Alternate name: 2EG J2023-0836
} 

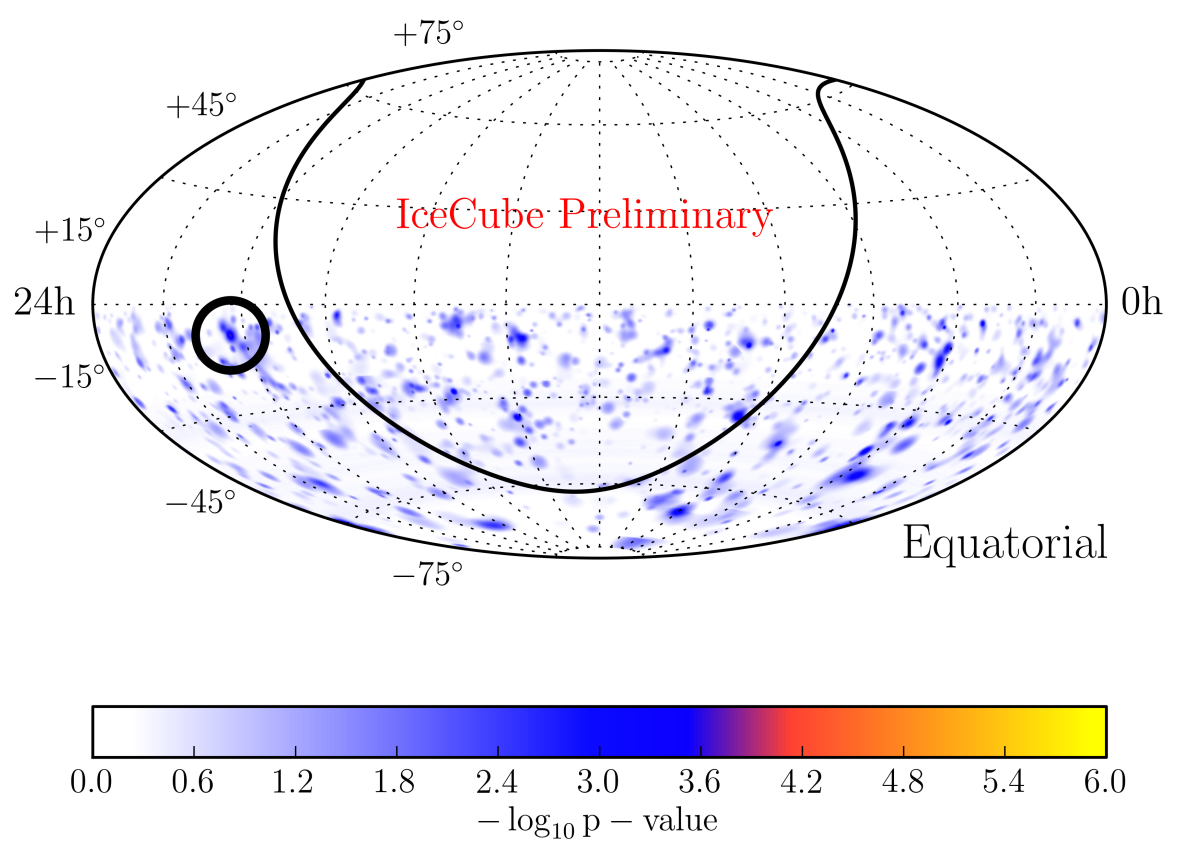

Figure 4: Pre-trial significance skymap in equatorial coordinates (J2000) showing the LESE analysis result on data from the first year of the full 86-string configuration of IceCube. The location of the Galactic plane is illustrated with a black solid line and the position of the most significant fluctuation is indicated with a black circle. The hottest spot is located at R.A. $305.2^{\circ}$ and dec. $-8.5^{\circ}$ with a pre-trial p-value of $1.6 \cdot 10^{-4}$. The corresponding post-trial p-value is $88.1 \%$.
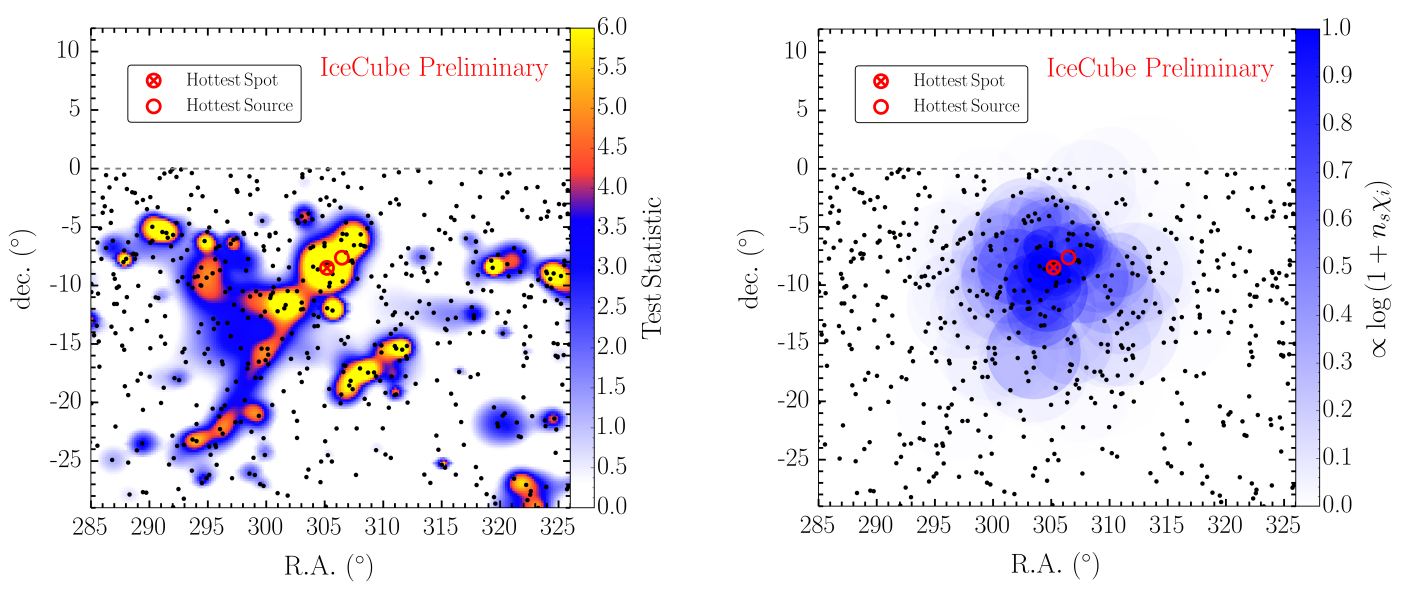

Figure 5: Zoom on the hottest spot (red circle + cross) and hottest source (red circle) in equatorial coordinates R.A. and dec. The reconstructed position of each event is shown in black dots. Left: The color transparency indicates the test statistic value at each pixel in the sky. Right: The radii of the circles are equal to the estimated angular uncertainty of each event. Color transparency indicate the strength of the individual likelihood weights $\log \left(1+n_{s} \chi_{i}\right)$ that contribute to the test statistic at the position and with the best fit parameters of the hottest spot. 
see figure 2. The limits of the five most significant sources are presented in table 1 along with the best fit parameters $n_{s}$ and $\gamma$. Note that we do not consider under fluctuations, i.e. for observed values of $\mathcal{T S}$ below the median of $\mathcal{T S}$ for the background-only hypothesis we report the corresponding median upper limit.

\section{Summary and Outlook}

No evidence of neutrino emission from point-like sources in the Southern Hemisphere at lowenergies $(100 \mathrm{GeV}$ - few $\mathrm{TeV})$ was found in the first year of data from the 86-string configuration of IceCube. The most significant spot in the sky has a post-trial p-value of $88.1 \%$. Further we tested the presence of signal from 96 sources in a pre-defined source list of known $\gamma$-ray emitters seen in ground-based telescopes. The results are consistent with the background-only hypothesis. The most significant source has a post-trial p-value of $14.8 \%$. Upper limits at $90 \%$ C.L. were calculated for these sources for a number of signal hypotheses, see figure 2 and table 1.

Three more years of IceCube data with the full configuration exist and are ready to be incorporated into this analysis. We expect the sensitivity to improve slightly faster than the square-root of time limit, due to the relatively low background rate. Further, we have plans of performing a search for extended point sources and for neutrino emission in the Galactic plane.

\section{References}

[1] W. Bednarek, G. F. Burgio, and T. Montaruli, New Astron.Rev. 49 (2005) 1.

[2] A. Kappes, J. Hinton, C. Stegmann, and F. A. Aharonian, Astrophys.J. 656 (2007) 870.

[3] M. D. Kistler and J. F. Beacom, Phys.Rev. D74 (2006) 063007.

[4] IceCube Collaboration, A. Achterberg et al., Astropart.Phys. 26 (2006) 155.

[5] IceCube Collaboration, R. Abbasi et al., Astropart.Phys. 35 (2012) 615.

[6] IceCube Collaboration, R. Abbasi et al., Nucl.Instrum.Meth. A700 (2013) 188.

[7] T. Neunhoffer, Astropart.Phys. 25 (2006) 220.

[8] IceCube Collaboration, M. Aartsen et al., JINST 9 (2014) P03009.

[9] J. Braun, J. Dumm, F. De Palma, C. Finley, A. Karle, et al., Astropart.Phys. 29 (2008) 299.

[10] K. M. Górski, E. Hivon, A. J. Banday, B. D. Wandelt, F. K. Hansen, M. Reinecke, and M. Bartelmann, The Astrophysical Journal 622 (2005), no. 2759.

[11] S. P. Wakely and D. Horan, TeVCat: An online catalog for Very High Energy Gamma-Ray Astronomy, in Proceedings, 30th International Cosmic Ray Conference (ICRC 2007), vol. 3, p. 1341, 2007.

[12] IceCube Collaboration, M. Aartsen et al., Medium-energy (few TeV - $100 \mathrm{TeV}$ ) neutrino point source searches in the Southern sky with IceCube, in PoS(ICRC2015)1056 these proceedings, 2015.

[13] IceCube Collaboration, M. Aartsen et al., Searches for Extended and Point-like Neutrino Sources with Four Years of IceCube Data, 2014. arXiv:1406.6757.

[14] J. Feintzeig, Searches for Point-like Sources of Astrophysical Neutrinos with the IceCube Neutrino Observatory. PhD thesis, University of Wisconsin, Madison, 2014.

[15] J. Neyman, Royal Society of London Philosophical Transactions Series A 236 (1937) 333. 